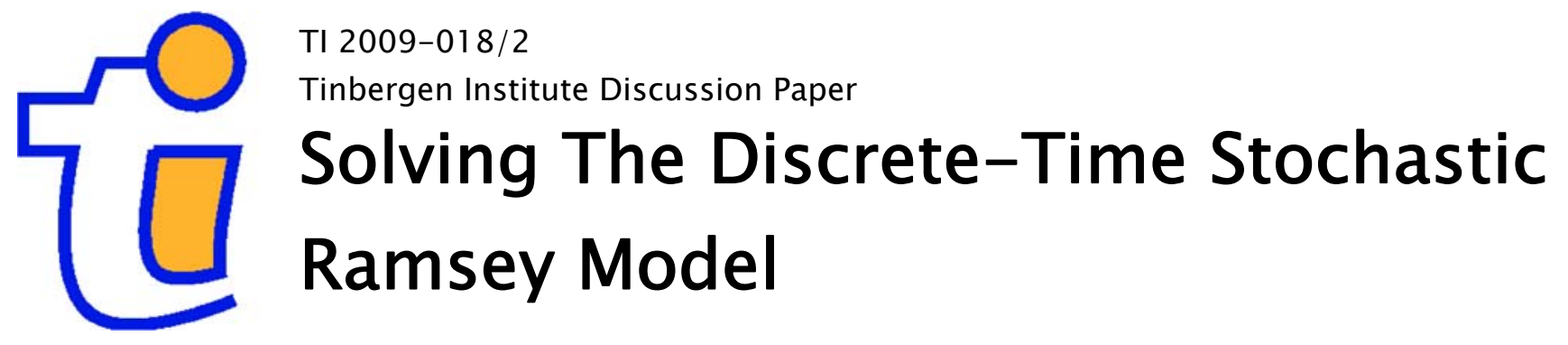

Chris Elbers

Faculty of Economics \& Business Administration, VU University Amsterdam, and Tinbergen Institute. 


\section{Tinbergen Institute}

The Tinbergen Institute is the institute for economic research of the Erasmus Universiteit Rotterdam, Universiteit van Amsterdam, and Vrije Universiteit Amsterdam.

Tinbergen Institute Amsterdam

Roetersstraat 31

1018 WB Amsterdam

The Netherlands

Tel.: +31(0)205513500

Fax: $+31(0) 205513555$

Tinbergen Institute Rotterdam

Burg. Oudlaan 50

3062 PA Rotterdam

The Netherlands

Tel.: + $31(0) 104088900$

Fax: $+31(0) 104089031$

Most TI discussion papers can be downloaded at http://www.tinbergen.nl. 


\title{
Solving the Discrete-Time Stochastic Ramsey Model
}

\author{
Chris Elbers \\ VU University Amsterdam
}

February 2009

\begin{abstract}
This note describes methods for solving deterministic and stochastic versions of the discrete-time Ramsey model of economic growth. We derive an iterative procedure for solving the Euler equation and apply it to an example adapted from Pan (2007).
\end{abstract}




\section{The deterministic Ramsey model}

Consider the following discrete-time intertemporal optimization problem

$$
\max _{\left\{k_{t}\right\}} \sum_{t=0}^{\infty} \beta^{t} u\left(c_{t}\right)
$$

subject to

$$
\begin{aligned}
c_{t} & =f\left(k_{t}\right)-k_{t+1} \\
k_{0} & =\text { given, }
\end{aligned}
$$

where $0<\beta<1$. The problem is to find a sequence of accumulated stock $\left\{k_{t}\right\}$ that will result in a consumption flow $\left\{c_{t}=f\left(k_{t}\right)-k_{t+1}\right\}$ that maximizes the discounted utility sum. We will not discuss here conditions under which this particular problem has a finite solution. Instead we will simply assume that a unique solution exists for every non-negative value of initial capital $k_{0}$.

The above problem is an example of the deterministic Ramsey model. It is stationary and it is not difficult to show that solutions satisfy

$$
k_{t+1}=\varphi\left(f\left(k_{t}\right)\right)
$$

for some 'investment function' $\varphi(w)$, where $\varphi(w)$ does not depend on $k_{0}$. Hence the problem may be rephrased as

$$
\max _{\varphi(.)} \sum_{t=0}^{\infty} \beta^{t} u\left(c_{t}\right)
$$

subject to

$$
\begin{aligned}
c_{t} & =f\left(k_{t}\right)-k_{t+1} \\
k_{t+1} & =\varphi\left(f\left(k_{t}\right)\right) \\
k_{0} & =\text { given }
\end{aligned}
$$

For ease of notation we will write $w=f(k) .{ }^{1}$ It is well-known ${ }^{2}$ that the optimal investment path $\left\{k_{t+1}\right\}$ satisfies the following intertemporal condition for $t=0,1,2, \ldots$ (the Euler equation):

$$
u^{\prime}\left(w_{t}-k_{t+1}\right)=\beta u^{\prime}\left(w_{t+1}-k_{t+2}\right) f^{\prime}\left(k_{t+1}\right)
$$

\footnotetext{
${ }^{1}$ In a typical application $w$ would be the sum of current income ( $y$, say) and the remaining value of the capital stock $((1-\delta) k): w=y+(1-\delta) k$.

${ }^{2}$ E.g., see Stokey and Lucas (1989).
} 
which shows that the real problem is to find $k_{1}$ : the rest of the sequence $k_{t}$ will then follow by repeatedly solving for $k_{t+1}$ in terms of $k_{t}$. The Euler equation leads to the following functional equation for $\varphi(w)$ :

For all $w \geq 0: u^{\prime}(w-\varphi(w))=\beta u^{\prime}(f(\varphi(w))-\varphi(f(\varphi(w)))) f^{\prime}(\varphi(w))$.

A solution to the stationary Ramsey problem converges to a steady state $k^{*}$ which (therefore) must satisfy the Ramsey rule

$$
1=\beta f^{\prime}\left(k^{*}\right) .
$$

One way to solve the Ramsey problem is to simply try various $k_{1}$ and see if the resulting path for $k_{t}$ gets close enough to the steady state ('shooting'). A reverse (and more practical) procedure is to linearize the dynamic path of $k_{t}$ around the steady state, make a very small step out of the steady state according to the linearized dynamics and then follow equation (1) backwards in time until a point near $k=0$ is reached, yielding $(w, \varphi(w))$ pairs. By perturbing the initial very small step out of steady state one can trace the graph (i.e. $(w, \varphi(w))$ pairs) of the function $\varphi(w) .^{3}$

Both the forward and backward shooting approach work fine but have the disadvantage that they do not generalize readily to a solution of the stochastic Ramsey model that we consider below. Among the methods that do generalize are the following:

'Binning' Binning reduces the infinite dimensional optimization problem to a finite dimensional one by aggregating $w$ and $k$ values in value brackets ('bins'). A so-called Bellman equation can be associated with the optimization problem stating that for given $k$ and corresponding $w=f(k)$ the program value $V(w)$ must satisfy

$$
V(w)=\max _{k^{\prime}} u\left(w-k^{\prime}\right)+\beta V\left(f\left(k^{\prime}\right)\right) .
$$

Solving this equation for $V(\cdot)$ is equivalent to solving the Ramsey problem. If values for $w$ and $k$ are restricted to finite sets $K=$ $(k(1), \ldots, k(n))$ and $W=(w(1), \ldots, w(m))$, then the function $V(\cdot)$ reduces to an $m$-vector $V=(V(w(1)), \ldots, V(w(m)))$ and $u\left(w-k^{\prime}\right)$ reduces to an $(n, m)$ matrix. The Bellman equation becomes

$$
\text { For all } j, V_{j}=\max _{i} u_{i j}+\beta V_{i} \text {. }
$$

\footnotetext{
${ }^{3}$ This is a discrete-time version of the so-called time elimination method. (E.g., Barro and Sala-i-Martin, 1995, "Appendix on mathematical methods".)
} 
Starting from an initial value of $V=0$, iteration of this equation yields a sequence of $V$ that converges monotonically to a solution of the discrete equation (since $\beta<1$ ). This is an approximation to the infinite dimensional problem. Likewise the argmax of the discrete equation readily leads to an approximation of the investment function $\varphi$. Elbers and Gunning (2007) use the binning approach to solve the stochastic Ramsey model to be discussed below.

Parametrization of the value/investment function. Solution by binning leads to step functions for $V$ and $\varphi$ which could be too ragged in practice. A way out is to fit a simple parametric family of functions to the discrete solution for $V$ or $\varphi$ and find the set of parameter values that best (in any given sense) solve the original Bellman equation.

Successive solution of the intertemporal equation. The previous solution method amounts to 'value function iteration'. Alternatively, a solution can be found by 'policy function iteration'. Omitting the time subscript equation (1) can be rewritten as

$$
u^{\prime}(w-k)=\beta u^{\prime}\left(f(k)-k^{\prime}\right) f^{\prime}(k)
$$

where $k^{\prime}=\varphi(f(k))$. Note that the above equation is the first order condition of the following maximization problem, where $k^{\prime}$ is now taken to be fixed.

$$
\max _{k} u(w-k)+\beta u\left(f(k)-k^{\prime}\right) .
$$

Let $k=\Phi\left(w, k^{\prime}\right)$ solve the above problem, then $\varphi(w)=\Phi(w, \varphi(f(\varphi(w))))$. This suggests the following iteration.

Start from a grid of values for $w=w_{1}, \ldots, w_{n}$ and initial values for $k_{1}^{\prime}, \ldots, k_{n}^{\prime}$. Solve problem (3) for each $w_{i}$ and put $\varphi_{i}=\varphi\left(w_{i}, k_{i}^{\prime}\right)$. Update the values for $k^{\prime}$ : ideally the updated values would be $k_{i}^{\prime}=$ $\varphi\left(f\left(\varphi_{i}\right)\right)$ but since the function $\varphi(\cdot)$ is not known we locate the grid values $w_{i}$ close to values of $f\left(\varphi_{i}\right)$ and interpolate/extrapolate the corresponding values for $\varphi_{i}$ to get new values of $k_{i}^{\prime}$. Solve problem (3) again for the new values of $k_{i}^{\prime}$ and repeat the procedure until the $\varphi_{i}$ no longer change.

A more formal description of the iterative process is as follows. Let $r$ indicate the iteration count, $\left\{\varphi_{i}^{r}\right\}$ the $r^{\text {th }}$ approximation to $\varphi\left(w_{i}\right)$, and $\hat{\varphi}^{r}(\cdot)$ the $r^{\text {th }}$ approximation to $\varphi(\cdot)$. In practice, the approximating function $\hat{\varphi}^{r}(\cdot)$ would be based on interpolation and extrapolation $(I P)$, 
linear or otherwise, of the current set of approximations $\left(w_{i}, \varphi_{i}^{r}\right)$, also taking into account that $\varphi(0)=0$ :

$$
\hat{\varphi}^{r}(\cdot)=I P\left(\left\{\varphi_{i}^{r}\right\}\right) .
$$

With this notation the next iterate is given by

$$
\varphi_{i}^{r+1}=\arg \max _{k} u\left(w_{i}-k\right)+\beta u\left(f(k)-\hat{\varphi}^{r}\left(f\left(\varphi_{i}^{r}\right)\right)\right),
$$

for $i=1, \ldots, n$. The converged value $\lim _{r \rightarrow \infty} \hat{\varphi}^{r}(\cdot)$ is the ultimate approximation to the investment function $\varphi(\cdot)$. Its accuracy will depend on the number of grid points $w_{i}$ and on the shape of the investment function $\varphi$.

\section{Generalization to stochastic versions of the Ramsey model}

The last three approaches mentioned in the previous section can all be generalized to solve stochastic versions of the Ramsey model. The first, binning, is discussed in Elbers and Gunning (2007); the second, parametrization, is an obvious variation of the first with the added advantage that (once the parametric form is chosen) it is not necessary to 'bin' the random shocks as well; the third is described in this section.

In the stochastic versions of the Ramsey model applied in Elbers and Gunning (2007) and Pan (2007) stock levels $k$ lead to random wealth $w=$ $f(k, s)$ that is divided into consumption and future stocks. Random shocks are denoted by $s$. The functional equation for the optimal investment function $\varphi$ becomes

$$
u^{\prime}(w-\varphi(w)) \equiv \beta E u^{\prime}(f(\varphi(w), s)-\varphi(f(\varphi(w), s))) \frac{\partial f}{\partial k}(\varphi(w), s),
$$

where $E$ denotes the expectation operator. The iterative scheme mentioned above changes accordingly: ${ }^{4}$

$$
\varphi_{i}^{r+1}=\arg \max _{k} u\left(w_{i}-k\right)+\beta E u\left(f(k, s)-\hat{\varphi}^{r}\left(f\left(\varphi_{i}^{r}, s\right)\right)\right) .
$$

In principle the expectation must be computed for every value of $k$ considered so that in practice the problem could be very costly to solve. Here

\footnotetext{
${ }^{4}$ This is based on the first-order condition for the solution of

$$
\max _{k} u(w-k)+\beta E u(s f(k)-\varphi(s f(\varphi(w)))),
$$

and subsituting $k=\varphi(w)$.
} 
we use a stochastic quasi gradient (SQG) method, ${ }^{5}$ which gradually approximates the expectation in parallel with the search for an optimal $k$, but in many cases we have seen that straightforward numerical integration (for each $k$ considered) or even crude approximations to the expectation work fine. ${ }^{6}$

\section{Examples $^{7}$}

\section{A deterministic Ramsey model}

Consider the following model which is similar to Pan (2007).

$$
\begin{aligned}
u(c) & =\log (c) \\
\beta & =0.9 \\
f(k) & =\left(1+0.208665 k^{0.363}\right)^{1.80992}+0.9 k \\
f(k, s) & =s_{y}\left(1+0.208665 k^{0.363}\right)^{1.80992}+0.9 k s_{k},
\end{aligned}
$$

where $s_{k}$ and $s_{y}$ are asset and income shocks. For the deterministic case the set of $w_{i}, \varphi_{i}$ values is listed in table 1 and graphed in figure 1 . The condition (2) is satisfied to within machine precision, both at the set of $w_{i}$ values and the midpoints $0.5\left(w_{i}+w_{i+1}\right)$ when optimal investment is computed by means of the converged function $\hat{\varphi}(\cdot)$.

\section{A stochastic Ramsey model}

For the stochastic case we choose $\left(s_{y}, s_{k}\right)$ to be lognormal, with unit expectation. The variances of $\log \left(s_{y}\right)$ and $\log \left(s_{k}\right)$ are equal to 0.25 , and the covariance is equal to 0.2 . The set of $w_{i}, \varphi_{i}$ values is listed in table 2 and graphed in figure 2. The ratio of the left-hand side and the right-hand side of condition (4) is tabulated for the set of $w_{i}$ values in table 3 and at midpoints in table 4 . This amounts to a precision within $1 \%$ of the true investment function. Finally, figure 3 graphs the deterministic and stochastic investment policies together. The two differ at higher values of $w$, the deterministic investment being slightly higher.

\footnotetext{
${ }^{5}$ See e.g., Wets (2005).

${ }^{6}$ For instance, in Pan (2008) each expectation is computed as the average over (the same) hundred draws from the shock-pair distribution. Pan (2008, Chapter 4, Appendix A) reports a very close approximation to the Euler equation..

${ }^{7}$ Mathematica code for the computations in this section is available from the author upon request.
} 


\section{References}

Barro, Robert J., and Xavier Sala-i-Martin. (1995) Economic Growth. New York: McGraw Hill.

Elbers, Chris, and Jan Willem Gunning. (2007) Growth and Risk: Methodology and Micro Evidence. World Bank Economic Review. 21 (1), pp $1-20$.

Pan, Lei. (2007) Risk Pooling through Transfers in Rural Ethiopia. Tinbergen Institute discussion paper 07-014/2.

Pan, Lei. (2008) Poverty, Risk and Insurance: Evidence from Ethiopia and Yemen. PhD Thesis VU University Amsterdam. Amsterdam: Thela Thesis.

Stokey, Nancy L., and Robert E. Lucas , Jr. (1989) Recursive Methods in Economic Dynamics. Cambridge: Harvard University Press.

Wets, Roger J-B. (2005) Stochastic Quasi-Gradient Methods. PowerPoint presentation, UCD February 15, 2005. 


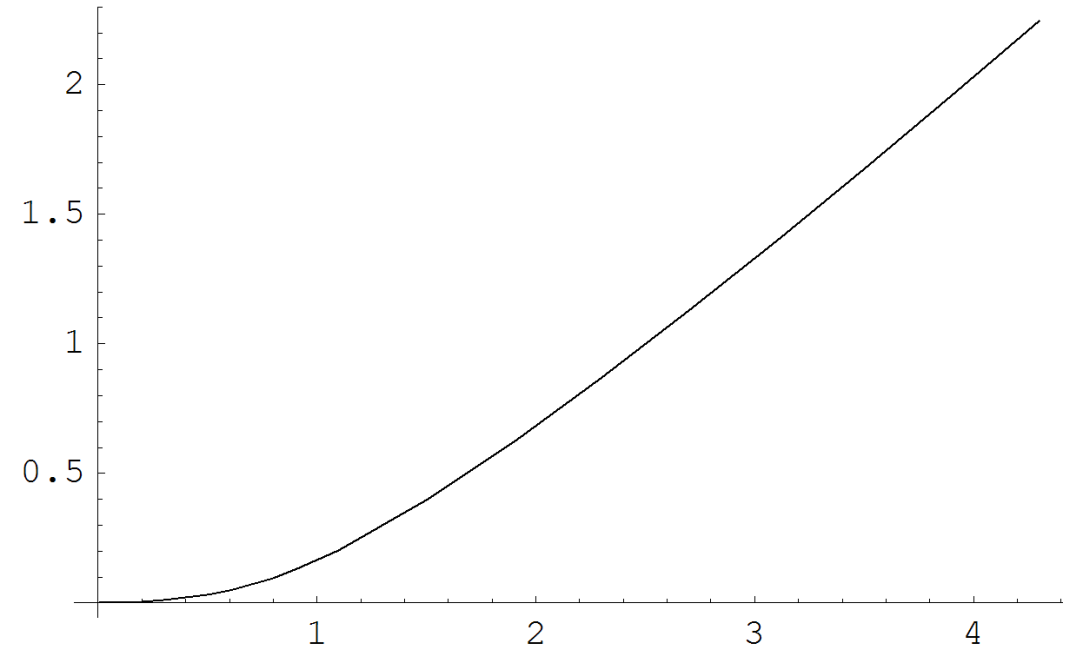

Figure 1: Investment function in the deterministic Ramsey model

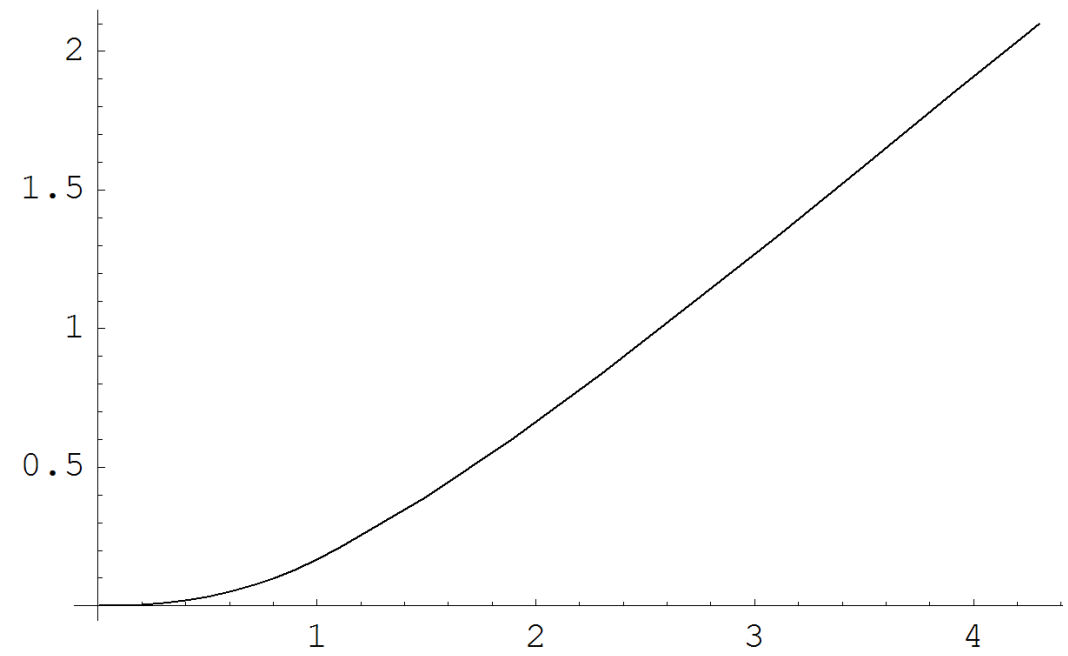

Figure 2: Investment function in the Stochastic Ramsey model 


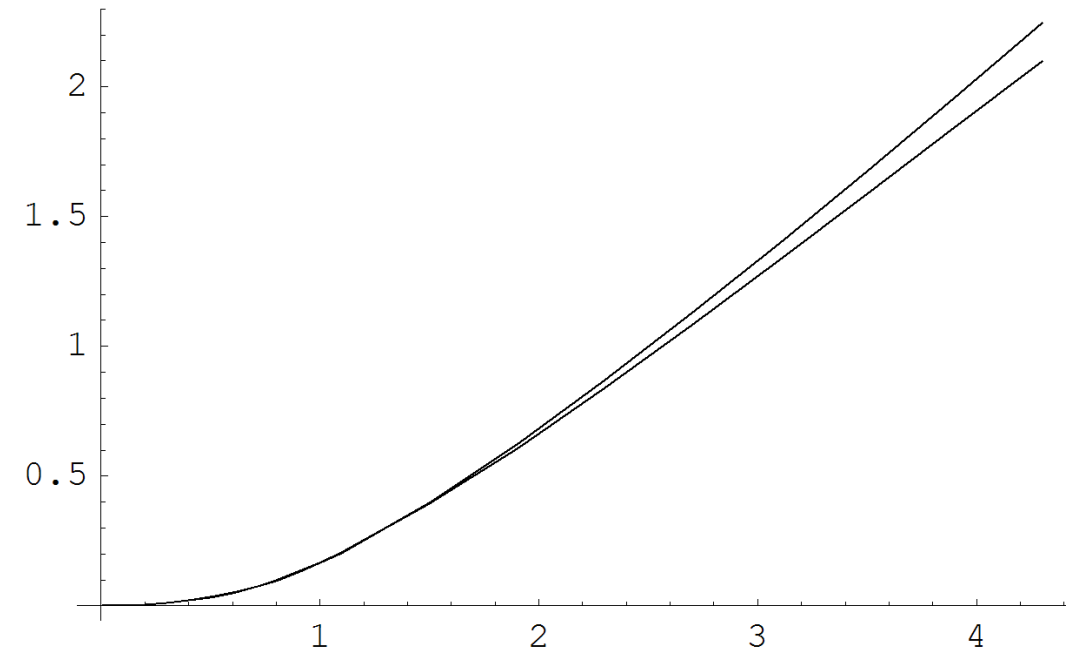

Figure 3: Investment function in the deterministic and stochastic Ramsey model. 


\begin{tabular}{ll}
$w$ & $\varphi$ \\
\hline 0.01 & 0.0000361472 \\
0.02 & 0.000108407 \\
0.03 & 0.000207159 \\
0.04 & 0.000329199 \\
0.05 & 0.000472882 \\
0.06 & 0.000637263 \\
0.07 & 0.00082179 \\
0.08 & 0.00102616 \\
0.09 & 0.00125022 \\
0.1 & 0.00149397 \\
0.2 & 0.00505967 \\
0.3 & 0.0109641 \\
0.4 & 0.0197818 \\
0.5 & 0.0321941 \\
0.6 & 0.0488639 \\
0.7 & 0.070295 \\
0.8 & 0.0967186 \\
0.9 & 0.128063 \\
1. & 0.164012 \\
1.1 & 0.204102 \\
1.5 & 0.396274 \\
1.9 & 0.622607 \\
2.3 & 0.868977 \\
2.7 & 1.12884 \\
3.1 & 1.39833 \\
3.5 & 1.67507 \\
3.9 & 1.95755 \\
4.3 & 2.24471
\end{tabular}

Table 1: Investment function in the deterministic Ramsey model 


\begin{tabular}{ll}
$w$ & $\varphi$ \\
\hline 0.01 & 0.0000672907 \\
0.02 & 0.000110104 \\
0.03 & 0.000210509 \\
0.04 & 0.00033423 \\
0.05 & 0.000480395 \\
0.06 & 0.000648734 \\
0.07 & 0.000834478 \\
0.08 & 0.00104408 \\
0.09 & 0.00127117 \\
0.1 & 0.00152136 \\
0.2 & 0.00518124 \\
0.3 & 0.0113158 \\
0.4 & 0.0204627 \\
0.5 & 0.0333911 \\
0.6 & 0.050716 \\
0.7 & 0.0727426 \\
0.8 & 0.0995818 \\
0.9 & 0.131158 \\
1. & 0.167132 \\
1.1 & 0.20666 \\
1.5 & 0.392224 \\
1.9 & 0.606471 \\
2.3 & 0.839481 \\
2.7 & 1.08268 \\
3.1 & 1.33161 \\
3.5 & 1.58754 \\
3.9 & 1.84329 \\
4.3 & 2.09772
\end{tabular}

Table 2: Investment function in the stochastic Ramsey model 


\begin{tabular}{ll}
$w$ & LHS(4)/RHS(4) \\
\hline 0.01 & 1.47171 \\
0.02 & 1.00108 \\
0.03 & 1.0011 \\
0.04 & 1.00024 \\
0.05 & 1.00029 \\
0.06 & 1.00125 \\
0.07 & 0.999379 \\
0.08 & 1.00026 \\
0.09 & 0.999515 \\
0.1 & 1.00012 \\
0.2 & 1.00012 \\
0.3 & 1.00146 \\
0.4 & 1.00041 \\
0.5 & 1.00047 \\
0.6 & 1.00071 \\
0.7 & 1.00039 \\
0.8 & 1.0001 \\
0.9 & 1.00039 \\
1. & 1.00123 \\
1.1 & 1.00145 \\
1.5 & 1.00147 \\
1.9 & 1.00112 \\
2.3 & 1.00269 \\
2.7 & 1.00295 \\
3.1 & 1.00161 \\
3.5 & 1.00204 \\
3.9 & 0.999681 \\
4.3 & 0.995176 \\
&
\end{tabular}

Table 3: Intertemporal condition in the stochastic Ramsey model at gridpoint values. 


\begin{tabular}{ll}
$w$ & LHS $(4) /$ RHS $(4)$ \\
\hline 0.015 & 0.870474 \\
0.025 & 1.00674 \\
0.035 & 0.999461 \\
0.045 & 0.99987 \\
0.055 & 0.999006 \\
0.065 & 0.999823 \\
0.075 & 1.00016 \\
0.085 & 1.00015 \\
0.095 & 1.00046 \\
0.15 & 0.995398 \\
0.25 & 0.999063 \\
0.35 & 0.999182 \\
0.45 & 0.99954 \\
0.55 & 0.999274 \\
0.65 & 0.999373 \\
0.75 & 0.999775 \\
0.85 & 0.99981 \\
0.95 & 0.999157 \\
1.05 & 0.998541 \\
1.3 & 0.998439 \\
1.7 & 0.999145 \\
2.1 & 0.998184 \\
2.5 & 0.997048 \\
2.9 & 0.997792 \\
3.3 & 0.998083 \\
3.7 & 0.998842 \\
4.1 & 1.00233
\end{tabular}

Table 4: Intertemporal condition in the stochastic Ramsey model, between gridpoint values. 